\title{
Re-thinking health professional education: 'think global, act local'-ensuring inclusivity and diversity of medical education in the new norm
}

\author{
Joynes, $V$. \\ United Kingdom
}

Healthcare education has long been a fast-moving discipline, but at no time in living memory have health care providers and their educators been faced with so many simultaneous challenges.

A long-overdue awakening of the lack of diversity in education resources and its impact on healthcare provided, and the requirement of healthcare providers and educators to keep going without pause in the midst of a global pandemic has challenged us as never before.

Nevertheless, if we are to draw positives from the last 12 months, we can recognise that the required pace of change within education has evidenced that some things that were previously considered implausible are now here. The almost complete removal of face to face teaching, the introduction of online assessments, and the early graduation of workforce are all new developments for many of us, across the globe. Co-operation across previously competitive Schools, regions and international borders has flourished in the face of shared adversity. Students, recognising new challenges, have stepped forward to volunteer in health services, teach peers, and co-produce new and innovative resources.

This talk will provide a point of reflection on these changes, asking whether these challenges have led us already to rethink what health professions education is, and should be in the future. It will question what long term lessons have been learned from the last 12 months, for ensuring we are ready to educate and graduate the global healthcare workforce of tomorrow. 